\title{
Real-World Characterization of Dimethyl Fumarate- Related Gastrointestinal Events in Multiple Sclerosis: Management Strategies to Improve Persistence on Treatment and Patient Outcomes
}

\author{
Jinny Min · Stanley Cohan · Enrique Alvarez - Jacob Sloane • \\ J. Theodore Phillips - Anneke van der Walt • Irene Koulinska • \\ Fang Fang · Catherine Miller · Andrew Chan
}

Received: November 27, 2018 / Published online: January 31, 2019

(C) The Author(s) 2019

\begin{abstract}
Introduction: Delayed-release dimethyl fumarate (DMF) is an effective treatment for multiple sclerosis (MS). Some patients experience gastrointestinal (GI) adverse events (AEs) that may lead to premature DMF discontinuation. This study characterized the impact of site-specific GI management strategies on the occurrence of GI events and discontinuation patterns.
\end{abstract}

Enhanced digital features To view enhanced digital features for this article go to https://doi.org/10.6084/ m9.figshare.7588421.

Electronic supplementary material The online version of this article (https://doi.org/10.1007/s40120019-0127-2) contains supplementary material, which is available to authorized users.

J. Min · I. Koulinska · C. Miller ( $₫)$

Biogen, Cambridge, MA, USA

e-mail: Catherine.miller@biogen.com

S. Cohan

Providence Multiple Sclerosis Center, Providence Brain and Spine Institute, Providence St Joseph

Health, Portland, OR, USA

E. Alvarez

Rocky Mountain Multiple Sclerosis Center,

University of Colorado, Aurora, CO, USA

J. Sloane

Beth Israel Deaconess Medical Center, Boston, MA, USA
Methods: Data on GI events and DMF persistence were retrospectively abstracted from medical records of patients treated with DMF in routine medical practice in the EFFECT study (NCT02776072). GI management strategies were assessed via a study site questionnaire. Discontinuation rates were analyzed according to counseling patterns.

Results: Of 826 DMF-treated patients at 66 sites, 809 from 65 sites were eligible for the GI analysis; of these, 27\% experienced GI AEs. Within 1 year of treatment, $14 \%(118 / 826)$ of patients discontinued DMF, 5\% (44/809) due to GI events. Most sites (92\%) reported that patients were very likely ( $>75 \%$ of the time) to be counseled about GI events at/before DMF treatment initiation and/or to be recommended that DMF be taken with food (86\%); $48 \%$ of sites reported to be very likely to recommend using

\section{J. T. Phillips \\ Department of Neurology, University of New \\ Mexico Health Sciences Center, Albuquerque, NM, USA}

A. van der Walt

Multiple Sclerosis Research Unit, Royal Melbourne Hospital, Parkville, VIC, Australia

\section{F. Fang}

Cytel Inc, Cambridge, MA, USA

A. Chan

Department of Neurology, Inselspital-Bern

University Hospital, University of Bern, Bern,

Switzerland 
symptomatic therapies for GI AEs. Lower discontinuation rates were reported at sites very likely versus not very likely ( $\leq 75 \%$ of the time) to (1) provide counseling; (2) provide specific details regarding GI events; or (3) recommend taking DMF with food, and/or using symptomatic GI therapies.

Conclusion: Counseling and other GI management strategies at initiation of DMF treatment appear to reduce the burden of GI events, and a variety of GI management strategies may improve DMF persistence.

Trial Registration: NCT02776072.

Funding: Biogen (Cambridge, MA, USA).

Keywords: Gastrointestinal events; Multiple sclerosis; Retrospective study; Tecfidera

\section{INTRODUCTION}

Delayed-release dimethyl fumarate (DMF) is an oral treatment option for patients with relapsing-remitting multiple sclerosis (RRMS). Upon oral administration, DMF is rapidly converted to its active metabolite monomethyl fumarate [1], which is then widely distributed throughout the body [2, 3]. DMF has demonstrated significant and sustained therapeutic efficacy across a range of clinical and radiologic measures in phase 3 clinical trials and in a long-term extension study, ENDORSE [4-6]. The overall safety and tolerability profile of DMF is favorable, although patients often experience gastrointestinal (GI) adverse events (AEs), such as diarrhea, nausea, abdominal pain, vomiting, and dyspepsia [7]. Clinical trials and community-based real-world studies have indicated that GI tolerability issues, if experienced, occur most frequently within the first 10-12 weeks of DMF treatment initiation, with the incidence decreasing over time. Thus, it is important that counseling patients regarding potential GI AEs occurs at or before treatment initiation [4, 6-10]. Although most treatmentemergent GI AEs are generally mild or moderate in nature [11], these events may lead to poor treatment compliance or premature DMF discontinuation for some patients.

In a pooled analysis of patients who received placebo $(n=771)$ or DMF $240 \mathrm{mg}$ twice daily $(n=769)$ in the 2-year, randomized, doubleblind, placebo-controlled phase 3 DEFINE and CONFIRM clinical trials, $31-40 \%$ of patients reported GI AEs, with $<1-3 \%$ of patients discontinuing DMF due to these events [7]. However, a multicenter, open-label, single-arm study (MANAGE) and other real-world, practice-based studies have reported discontinuation rates of $10-29 \%$ for all AEs and 7-14\% for GI-specific AEs [8, 12, 13].

As of July 31, 2018, > 340,000 patients have received DMF treatment, representing $>625,000$ patient-years of exposure [14]. Therefore, real-world DMF data can offer important, potentially more generalizable, insights into management strategies for GI AEs. In order to capture the levels and types of consensus on best practice management strategies from DMF prescribers, a Delphi study was conducted among North American clinicians [10]. Clinicians completed two rounds of questionnaires developed by a steering committee; consensus in round 2 was considered to be attained if $\geq 70 \%$ of respondents agreed on a particular strategy. The Delphi consensus on effective GI management strategies, as well as a preliminary report suggesting that individual patient coaching leads to decreased avoidable therapy discontinuation due to GI AEs [10, 15], was utilized to guide this research [10].

The EFFECT study (NCT02776072) was undertaken to evaluate the comparative effectiveness of DMF in patients with RRMS in both a clinical practice setting and in a subset of newly diagnosed patients. As part of the EFFECT study, a GI analysis was designed to further investigate (1) the strategies to manage and mitigate DMFassociated GI events in the real-world setting and (2) the type of GI AEs occurring in a realworld setting, GI AEs leading to treatment discontinuation, and the frequency of GI event management strategies used in patients treated with DMF [16].

\section{METHODS}

\section{Study Design and Patients}

The EFFECT study was a global, multicenter, medical chart abstraction study in patients with 
RRMS treated with select disease-modifying therapies [DMTs; i.e., DMF, glatiramer acetate (GA), teriflunomide, or fingolimod] according to routine clinical practice [Electronic Supplementary Material (ESM) Fig. S1]. Key patient inclusion criteria were written informed consent; age $\geq 18$ years; diagnosis of RRMS per 2010 McDonald criteria [17]; DMF treatment initiation after January 2011 with $\geq 12$ months of follow-up data available following DMF initiation; treatment naive or have had only one prior DMT [interferon (IFN) or GA]; and multiple sclerosis relapse history for up to 3 years. Patients were included irrespective of total time on treatment. Key exclusion criteria were diagnosis of a progressive form of MS before or during data collection; prior treatment with a DMT other than IFN or GA; treatment with any formulations of DMF or compounded fumarates prior to DMF treatment initiation; and concurrent enrollment in any interventional clinical trial of an investigational product during the time of medical chart abstraction.

The GI analysis was conducted at sites that abstracted data from patients receiving DMF treatment. Sites were selected based on feasibility assessment of willingness and capability for participation in the study. Factors such as approximate number of patients treated with MS therapies, site start-up timelines, ability to retrospectively identify eligible medical records, and resources available for chart abstraction were considered. Chart abstraction occurred from May 2016 through January 2017.

Medical charts were reviewed at a single time point, and patient information was captured and managed by study sites on web-based electronic case report forms. Data were collected for patients receiving DMF for selected GI AEs and included type of event, date of onset, duration of event, concomitant treatment received, and any interruption, dose reduction, or discontinuation of DMF treatment. Physicians or their designees completed a structured, site-level questionnaire to report the likelihood of executing strategies for mitigating and/or managing GI events that may be experienced by DMFtreated patients. Responses from the questionnaire were collected to evaluate GI management practices (ESM Fig. S1) using the scale described below and were reported in the aggregate as frequencies.

\section{Outcomes}

The GI analysis endpoints were the proportion of DMF discontinuations related to selected GI events overall, by site, and by patient demographics, as well as the frequency of each strategy used for mitigating and managing GI events. A post hoc analysis assessed GI events and discontinuation rates according to survey responses to evaluate the impact of counseling on clinical outcomes.

\section{Questionnaire to Evaluate GI Management Practices}

The structured questionnaire for evaluating GI management practices consisted of a total of 26 items assessing the site characteristics, respondent type (e.g., neurologist, nurse practitioner), resources used for counseling, counseling habits (as outlined in the questionnaire, see ESM Appendix S1), and GI AE mitigation techniques. The questionnaire was built to assess the likelihood that sites were utilizing the techniques recommended by physicians using the Delphi consensus methodology to manage or mitigate GI events with DMF [10]. The questionnaire was developed in collaboration with physician and nurse healthcare providers (HCPs) and was piloted in a separate group of HCPs to assess for clarity and comprehension of the individual questions and to assess the duration of time required to complete the questionnaire.

Each site completed one questionnaire, responding to as many questions as possible. Sites were asked to describe their practice center, including size, type, and available staff. Sites also indicated the likelihood of various GI management and counseling strategies using a scale as follows:

- Not very likely $(\leq 75 \%)$

- Never $(0 \%)$

- Unlikely (>0-25\%)

- Somewhat likely (> 25-75\%)

- Very likely (> 75\%) 


\section{Statistical Analysis}

All analyses were performed using $\mathrm{SAS} \circledast{ }^{\circledR}$ version 9.2 or higher (SAS Institute, Cary, NC, USA). Descriptive analyses corresponding to the specific research objectives were performed to characterize the GI analysis population. Continuous variables are reported as number of patients, mean, standard deviation (SD), median, minimum, and maximum where appropriate. Categorical variables are summarized as number of patients and percentage.

Data were summarized for (1) select GI events and GI events leading to DMF discontinuation overall and over time, (2) each GI management strategy as reported in the GI questionnaire, and (3) the site-level discontinuation rates according to counseling patterns.

\section{Standard Protocol Approvals, Registrations, and Patient Consent}

The protocol was approved by the relevant Institutional Review Board for each study site. All procedures performed in studies involving human participants were in accordance with the ethical standards of the institutional and/or national research committee and with the 1964 Helsinki declaration and its later amendments or comparable ethical standards. Informed consent was obtained from all individual participants included in the study. The study was conducted in accordance with the Declaration of Helsinki, International Conference on HarmonizationGood Clinical Practice, and all applicable laws and regulations. A waiver of informed consent, based on non-personally identifiable retrospective data collection and minimal risk, was at the discretion of the individual Institutional Review Boards or Ethics Committees for each site. When required, participants provided written informed consent before study enrollment.

\section{RESULTS}

\section{Patients and Sites}

Overall, 826 DMF-treated patients were enrolled at 66 sites (Table 1), with patient recruitment per site ranging from 1 to 107 patients (median 7). Mean (SD) age was 44 (12) years; 76\% (624/ $826)$ of patients were female; $57 \%(467 / 826)$ of patients were from the USA (Region 1); and 32\% $(265 / 826)$ were treatment naive. Of the patients with a prior MS treatment, $76 \%(393 / 515)$ had received IFN beta. A total of $14 \%(118 / 826)$ of patients discontinued treatment with DMF, most commonly due to tolerability issues [9\% (71/826 patients)].

\section{GI Analysis}

A total of 809 patients from 65 sites who completed the GI questionnaire were included in the GI analysis. In the first 12 months of treatment, $27 \%$ (216) of patients had at least one GI event recorded in their medical charts. The most commonly reported GI events were nausea [11\% (87 patients)], diarrhea [9\% (74)], abdominal pain [6\% (51)], vomiting [5\% (43)], upper abdominal pain [2\% (20)], abdominal discomfort [2\% (19)], and dyspepsia [2\% (15)]. The majority $(81 \%)$ of GI events occurred within the first 5 weeks of DMF treatment initiation (Fig. 1). However, only 5\% (44 patients) discontinued DMF due to GI events, with nausea $[3 \%(21)]$ being the most common event leading to discontinuation, followed by diarrhea [2\% (19)], abdominal pain [2\% (15)], and vomiting [2\% (13)]. Similar to the onset of GI events, the majority of events leading to discontinuation occurred in the first 5 weeks of therapy $(92 \%)$.

Titration schedules were recorded in the medical charts for 727/809 patients; data were missing for 82 patients. Approximately 50\% (331/727) of patients titrated their DMF dose for 1 week, 20\% (151/727) titrated it for 2-4 weeks, and $<10 \%(59 / 727)$ titrated it for $>4$ weeks. No pattern of discontinuation was identified when the data were stratified by titration schedule. However, the number of patients with no titration schedule reported in their medical records $(186 / 727)$ or with missing data impacted the evaluable sample size and the ability to interpret the data. Similarly, of the 216 patients with $\geq 1$ GI event, only $4 \%$ received dosage modification and $11 \%$ received symptomatic 
Table 1 Baseline characteristics and patient disposition

\begin{tabular}{|c|c|}
\hline Characteristic & $\begin{array}{l}\text { DMF-treated patients } \\
(n=826)\end{array}$ \\
\hline Age, median (min, $\max )$, years & $44(18,73)$ \\
\hline Age, mean (SD), years & $44(12)$ \\
\hline Female & $624(76)$ \\
\hline \multicolumn{2}{|l|}{ Region $^{a}$} \\
\hline Region 1 & $467(57)$ \\
\hline Region 2 & $320(39)$ \\
\hline Region 3 & $39(5)$ \\
\hline Any prior MS treatment ${ }^{b}$ & $515(63)$ \\
\hline IFN beta & $393(48)$ \\
\hline GA & $115(14)$ \\
\hline Other $^{\mathrm{c}}$ & $7(<1)$ \\
\hline Treatment naive & $265(32)$ \\
\hline \multicolumn{2}{|c|}{ Reasons for discontinuing prior MS treatment ${ }^{\mathrm{b}, \mathrm{d}, \mathrm{e}}$} \\
\hline Tolerability & $244(30)$ \\
\hline Efficacy & $165(20)$ \\
\hline Adverse events & $14(2)$ \\
\hline Patient preference & $130(16)$ \\
\hline Other & $53(6)$ \\
\hline $\begin{array}{l}\text { Discontinued DMF by } 1 \text { year, } \\
n(\%)\end{array}$ & $118(14)$ \\
\hline \multicolumn{2}{|l|}{ Reasons for discontinuing $\mathrm{DMF}^{\mathrm{d}}$} \\
\hline Tolerability & $71(9)$ \\
\hline Efficacy & $19(2)$ \\
\hline Safety & $16(2)$ \\
\hline Patient preference & $14(2)$ \\
\hline Other & $11(1)$ \\
\hline
\end{tabular}

Values in table are presented as a number with the percentage in parenthesis, unless noted otherwise

$D M F$ delayed-release dimethyl fumarate, $G A$ glatiramer acetate, IFN interferon, max maximum, min minimum, $M S$ multiple sclerosis

a Region 1: USA; Region 2: Argentina, Australia, Canada, France, Germany, Italy, Spain, Switzerland, UK; Region 3: Croatia, Czech Republic, Hungary

b Based on 816 patients with data available

c Five patients previously used a chronic MS steroid, one patient received natalizumab, and one patient received rituximab

d More than one reason for discontinuing prior MS treatment was permitted; data were collected retrospectively

e Reasons for discontinuation were based on clinical judgement; no formal criteria were provided therapy. Therefore, it was not possible to perform additional analyses due to the small number of evaluable patients.

Discontinuation rates due to GI events varied by region. Regions were defined on the basis of geography, as well as by type of healthcare system and access to health care in each country. Discontinuation rates were $7.1 \%(32 / 451)$ in Region 1 (USA), 3.4\% (11/320) in Region 2 (Argentina, Australia, Canada, France, Germany, Italy, Spain, Switzerland, UK), and 2.6\% (1/38) in Region 3 (Croatia, Czech Republic, Hungary).

\section{Patient Management Strategies: Questionnaire Results}

\section{Respondent and Site Characteristics}

Questionnaire respondents were physicians $(48 \%)$, registered nurses (15\%), nurse practitioners $(14 \%)$, physician assistants $(2 \%)$, and other $(22 \%$; included clinical research coordinators, research assistants, and study coordinators). Respondents characterized their site type as nonhospital-based community practice (45\%), hospital-based community practice $(28 \%)$, academic setting (18\%), or other $(9 \%)$. They characterized their practice size as $\leq 250$ patients (25\%), 251-500 patients (14\%), 501-1000 patients (26\%), or $>1000$ patients (35\%).

\section{Site-Reported GI Management and Mitigation Practices}

Most sites [86\% (54/63)] were very likely to recommend that DMF be taken with food or that specific diets be followed, such as high fat, high protein, or high starch (Fig. 2a). Additionally, $48 \%(30 / 62)$ of sites were very likely to recommend the use of symptomatic therapies for GI AEs. The most frequently recommended symptomatic therapies included proton pump inhibitors, secretion blockers, and antidiarrheal medications (Fig. 2b).

\section{Site-Reported Counseling Practices}

Most sites [92\% (58/63)] reported that patients were very likely to be counseled orally regarding GI events at or before the initiation of DMF 
(a) GI AES

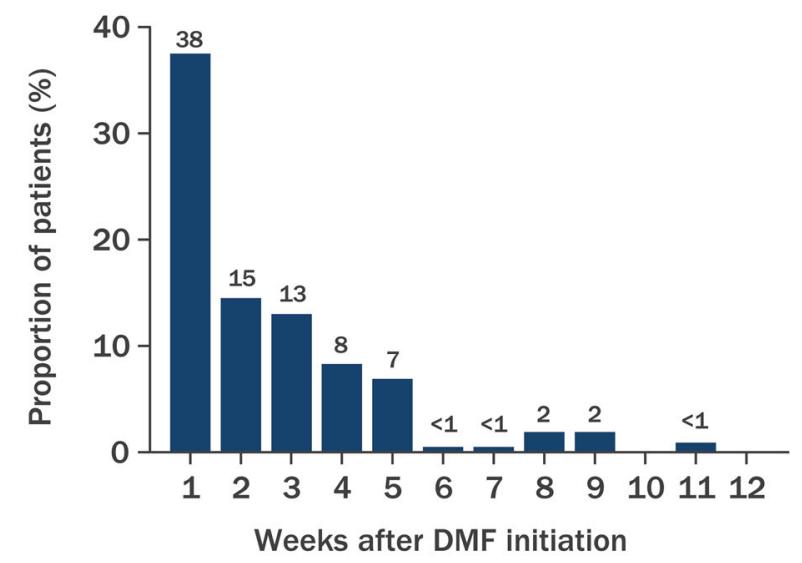

(b) Gl events leading to discontinuation

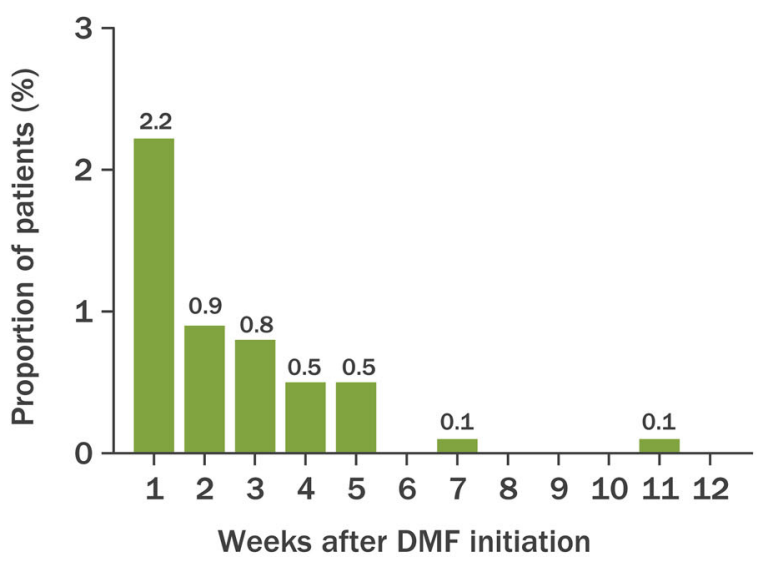

Fig. 1 Incidence of gastrointestinal $(G I)$ adverse events $(A E s)$ over time. The numbers above bars are the proportion (in percentage) of patients with GI AEs. DMF Delayed-release dimethyl fumarate

(a) Types of foods

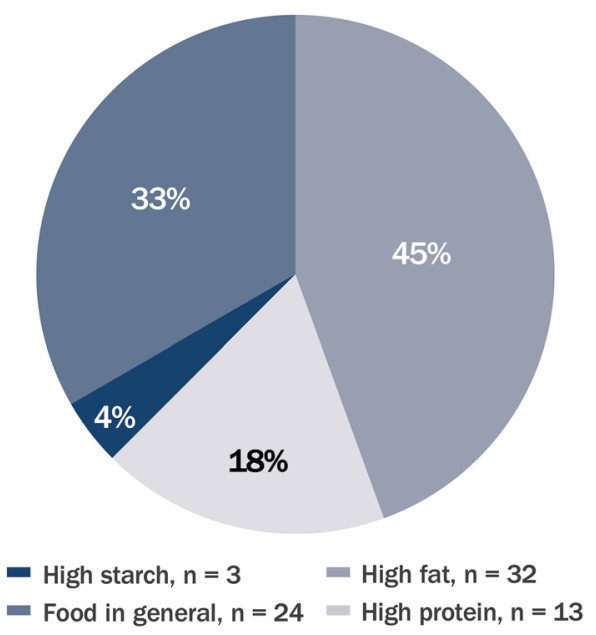

(b) Classes of medication(s)

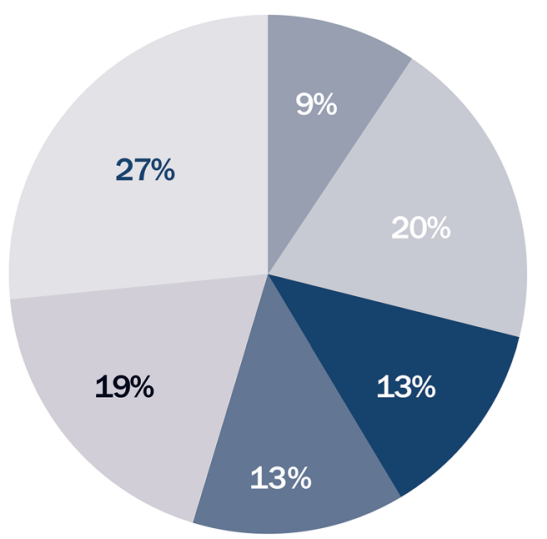

\footnotetext{
- Bismuth subsalicylate, $\mathrm{n}=16 \quad$ Proton pump inhibitors/

- Centrally acting antiemetics, $n=17 \quad$ antisecretion blockers, $n=34$

- Antibloating/anticonstipation, $n=12$ Other, $n=24^{a}$

- Antidiarrheal, $\mathrm{n}=25$
}

Fig. 2 Most commonly recommended types of foods (a) and classes of medication(s) (b) for GI event mitigation. Sites were instructed to select all that applied. $n$ Number of sites (total number of sites 65 ). ${ }^{\text {a Other }}$

treatment (Fig. 3), and 34\% (22/64) of sites were very likely to utilize a member of the healthcare team, in addition to the prescriber, to counsel patients at or before DMF treatment initiation (Fig. 3). In the event the prescriber was unavailable to provide counseling at or before DMF treatment initiation, $19 \%(12 / 63)$ of sites were very likely to utilize another member of the healthcare team to counsel patients. The other classification included antacid medications (e.g., calcium carbonate) $(n=22)$; histamine 2 blocker $(n=1)$, and nonsteroidal anti-inflammatory drugs $(n=1)$

HCPs providing counseling included registered nurses, physicians other than the prescribing physician, nurse practitioners, and physician assistants. Most sites [83\% (52/63)] were very likely to provide patients with specific details of GI events (timing of onset, incidence, severity, and duration); $44 \%(28 / 64)$ of sites were very likely to provide patients with information about GI events in written form (handwritten, printed, 




Fig. 3 Counseling practices regarding GI event associated with DMF treatment, reported by sites. Very likely indicates counseling for $>75 \%$ of the time. Not all sites responded to all questions $[n=63$ for counseled; $n=64$ for counseled by another healthcare provider $(H C P)$ in addition to the prescriber; $n=63$ for counseled by

emailed, and online), and 92\% (59/64) of sites instructed patients to contact the office if they experienced GI events. Seventy-eight percent (49/63) of sites were very likely to advise patients to titrate the dose of DMF over the first week of treatment to prevent GI events, but only $41 \%$ (26/63) of sites were likely to advise patients to temporarily reduce the dose of DMF if the patient experienced GI events; only 26\% (14/53) of sites were very likely to advise patients to temporarily reduce the dose of DMF to $120 \mathrm{mg}$ twice daily, as per approved package labeling, if they experienced GI events; and only 13\% (8/63) of sites were very likely to advise patients to temporarily interrupt the dose of DMF. Most sites [86\% (54/ 63)] were very likely to recommend food as therapy; $48 \%$ (30/62) of sites were likely to recommend symptomatic therapy. Sites that were likely to counsel patients orally also were likely to indicate that written information was available $[42 \%(27 / 65)]$, to recommend food [77\% (50/ $65)]$, and to recommend concomitant symptomatic therapy [45\% (29/65)].

\section{Discontinuation Rates According to Counseling Patterns: Questionnaire Results}

In a descriptive analysis, lower discontinuation rates were reported at sites that were very likely,

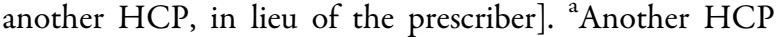
in addition to the prescriber could include a registered nurse, physician, nurse practitioner, physician assistant, or pharmacist. ${ }^{b} \mathrm{HCP}$ in lieu of the prescriber could include a registered nurse, physician, nurse practitioner, physician assistant, or pharmacist

versus sites that were not very likely, to have a member of the healthcare team provide counseling in addition to or in lieu of the prescribing physician, provide specific details about GI events, recommend taking DMF with food, and/ or use symptomatic GI therapies (Fig. 4). Discontinuation rates were similar at sites that were very likely to provide counseling in the form of written information versus sites that were not very likely to do so (Fig. 4). The sites that utilized written information in their handouts included GI management strategies identified in the Delphi study (ESM Appendices S2 and S3).

\section{DISCUSSION}

In this GI analysis of DMF-treated patients, the majority of GI AEs and GI-related discontinuations in this study occurred within the first month of treatment initiation, stressing the importance of preparing the patient for possible DMF-related GI events and the need for early intervention. Similarly to the single-arm studies MANAGE and TOLERATE, the incidence of GI events was highest in the first month of DMF treatment $[8,18]$. Unfortunately, due to the retrospective nature of this study, based on data collection via chart abstraction, we were not able to determine the prevalence or duration of 


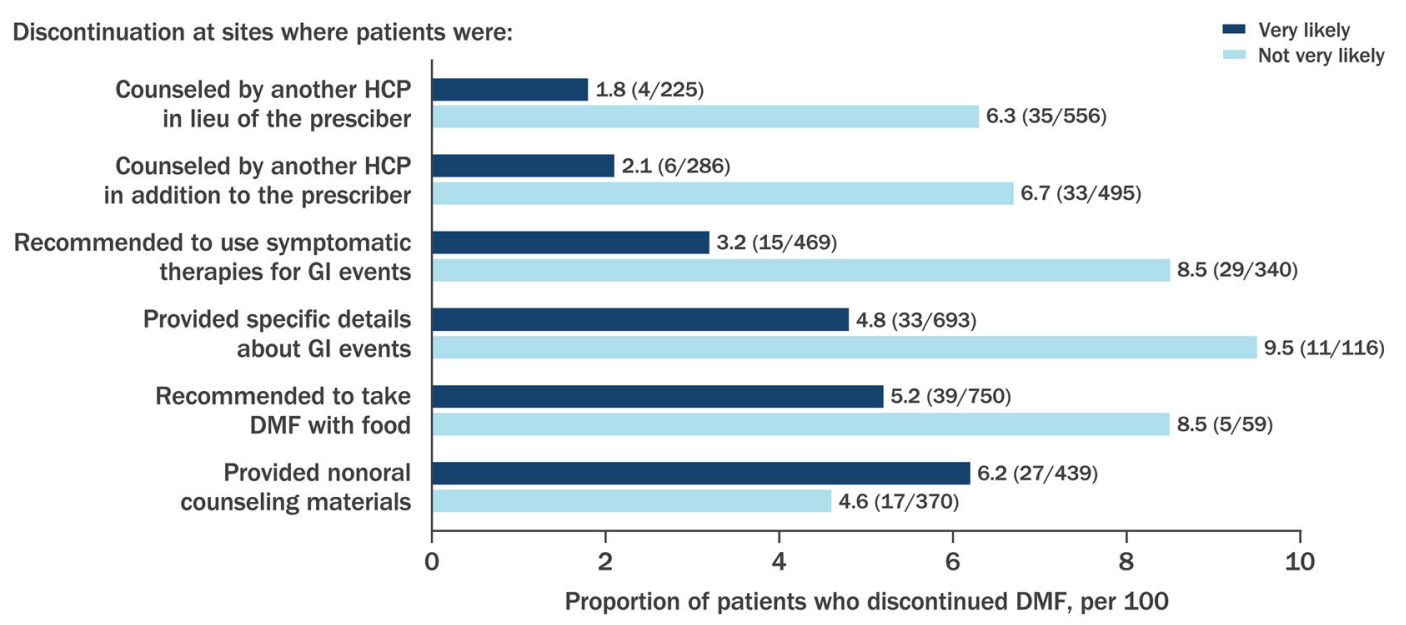

Fig. 4 Proportion of patients who discontinued DMF by site frequency of GI management strategy implementation. Specific details of GI event included information on timing of onset, incidence, severity, and duration. Nonoral counseling materials included handwritten, printed, emailed, online, or other information. Sites could select more than one option. Not all sites responded to all

AEs because recurrent events may not have been captured and AE "stop dates" are frequently not reported in medical charts or listed as unknown. A future prospective clinical trial in which prevalence and duration of AEs are analyzed would be informative.

The incidence of GI AEs (27\%) and the proportion of patients discontinuing treatment as a result of GI AEs (5\%) in our analysis was somewhat lower than has been reported in other realworld studies, but similar to what was observed in controlled clinical trials $[4,6,11]$. The data from the site survey responses indicate a high likelihood that the patients in this study were counseled about GI symptoms associated with DMF treatment. It is hypothesized that the implementation of GI management and mitigation strategies mimics the clinical trial experience for the patient and is improving as experience in the use of DMF increases. DMF was approved for use by the US Food and Drug Administration in March 2013, followed by the European Medicines Agency in January 2014. Thus, of the patient charts abstracted for this study, more patients initiated DMF during the period 2014-2015 [65\% (528/809)] versus previous years $[35 \%(281 / 809)]$. There were fewer questions; sites may have been in the very likely and/or not very likely category for each of the mitigation strategies depending on their response in the questionnaire. Very likely, $>75 \%$ of the time; not very likely, $0-75 \%$ of the time. Number of discontinuations/number of patients who received the management strategy described is shown in the figure (proportions are indicated along the $X$-axis)

discontinuations due to GI AEs among patients initiating DMF treatment in the latter half of the recruitment period versus the initial half of the recruitment period $[4.2 \%(22 / 528)$ vs. $7.8 \%$ $(22 / 281)]$, which supports the hypothesis that experience with DMF is resulting in improved practice management over time. Another explanation for the lower number of discontinuations due to GI AEs in our analysis compared to those reported in other real-world analyses $[8,12,13]$, which may also relate to the timing of DMF initiation, is that the majority of patients enrolled in this study initiated therapy during the latter half of the study when prescribers had a better understanding of how to manage patients with GI events using tools like the Delphi consensus paper as references [10]. In general, sites that were more likely to frequently utilize these strategies had lower rates of discontinuation due to GI AEs in this study, although only discontinuation rates, not adherence to treatment, were specifically assessed. Overall discontinuation rate was utilized as a surrogate for adherence to treatment, which does not necessarily mean the patient received the full dose; for example, treatment could have decreased to one dose per day to enable the 
patient to better tolerate the GI effects. Dose modifications were not measured in this study, but they are an important variable to assess in future research. Finally, another inherent limitation of this study, due to its design, is the possibility of under-reporting of events collected in this study, as the data are reliant upon accuracy of the medical charts.

Lower discontinuation rates were observed at sites that were very likely, versus sites that were not very likely, to have an HCP, in addition to or in lieu of the prescriber, counseling patients, providing specific details about GI events, recommending taking DMF with food, or recommending using symptomatic therapy. Therefore, providing this type of specific guidance to patients may be most helpful to reducing discontinuations due to GI events. Of note, only a small percentage of patients were recorded actually using symptomatic therapy or having a modified dose titration schedule in the patientlevel chart data. Due to inherent limitations in the study design, this value may be under-reported because the majority of symptomatic therapies are over-the-counter therapies or because dose titration instructions may have been given verbally to some patients and not recorded in the medical chart data. Additionally, this study did not demonstrate that use of written information as a counseling strategy was effective at reducing site-level discontinuation rates. It is speculated that written counseling may have been offered instead of (rather than a supplement to) oral counseling. Other studies have shown written forms of counseling may be an effective part of the DMF GI management strategy $[15,19]$.

The EFFECT study observed varying discontinuation rates among the different regions. Potential reasons for the observed variations in discontinuation rates include differences in regional practices of implementing GI management strategies and variation in years of experience in prescribing DMF. As part of a post hoc analysis, it was observed that the incidence of GI adverse events in Region 1 [29\% (129/ 451)] versus combined Region 2/3 [24\% (87/ $358)]$ were similar in this study, yet discontinuation rates were strikingly different in Region 1 [7\% (32/451)] versus combined Region $2 / 3$ [3\% $(12 / 358)]$. Consistent with the analysis for the entire population, similar patterns of lower discontinuation rates for patients initiating therapy in more recent years were also observed when stratified by region. One hypothesis for the difference in discontinuation rates despite a similar incidence of AEs is that the management and mitigation strategies implemented by these regions drive the differences in discontinuations due to GI AEs associated with DMF treatment. Another hypothesis is that limited access to a wider range of MS treatment alternatives in particular countries may result in lower discontinuation rates irrespective of side effects. Other inherent country differences that may influence patient tolerability to DMF and willingness to tolerate side effects, such as diet, availability of over-the-counter symptomatic therapies, exercise, meal timing, and cultural norms that discourage the reporting of side effects, should be noted as well, as supported by other real-world studies from several countries that have reported differences in overall and GIrelated discontinuation rates $[12,13,18,20]$.

In summary, DMF is efficacious for the treatment of RRMS, and oral administration is a convenient dosing form for patients. However, for patients to optimize the efficacy benefits of DMF they must overcome potential initial tolerability hurdles, including DMF-related GI AEs. GI counseling and management strategies implemented early in the treatment course may be critically important in driving patients to reach this goal.

\section{CONCLUSIONS}

The results of this study suggest that the use of a variety of GI counseling and management strategies may improve persistence on DMF therapy and increase the likelihood that patients can optimize the efficacy benefits of long-term DMF treatment.

\section{ACKNOWLEDGEMENTS}

The authors wish to thank the patients who participated in this study, which allowed this research to be possible. 
Funding. This study was sponsored by Biogen (Cambridge, MA, USA). All authors had full access to all of the data in this study and take complete responsibility for the integrity of the data and accuracy of the data analysis. Biogen funded the article processing charges.

Medical Writing, Editorial, and Other Assistance. Writing and editorial support for the preparation of this manuscript was provided by Excel Scientific Solutions (Southport, CT, USA). Funding was provided by Biogen.

Authorship. All named authors meet the International Committee of Medical Journal Editors (ICMJE) criteria for authorship for this article, take responsibility for the integrity of the work as a whole, and have given their approval for this version to be published.

Disclosures. Jinny Min was a contractor for Biogen at the time of study analysis and is currently an employee of Rhythm Pharmaceuticals. Stanley Cohan has received consulting fees from advisory boards and from steering committees for Biogen, Genzyme, and Novartis; speaker fees from Acorda, Biogen, Genentech-Roche, Novartis, and SanofiGenzyme; research grants from Biogen, Genentech-Roche, MedDay, Novartis, and Sanofi-Genzyme, and speakers bureau for Biogen, GenentechRoche, and Sanofi-Genzyme. Enrique Alvarez has received consulting fees from Actelion, Biogen, Celgene, EMD Serono, Genentech, Genzyme, Novartis, and TG Pharmaceuticals; research funding from Acorda, Biogen, Genentech, Novartis, and the Rocky Mountain MS Center. Jacob Sloane has received consulting fees from Biogen, Genentech, Genzyme, and Teva; research grants from Biogen and Genzyme. J. Theodore Phillips has received consulting or speaking fees from Acorda, Biogen, Genzyme, and TG Pharmaceuticals. Anneke van der Walt has received consulting fees from advisory boards for Genzyme, Merck, and Novartis; travel honoraria from Biogen, Merck, Novartis, and Teva. Fang Fang is an employee of Cytel Inc. Irene Koulinska is an employee of and holds stock/stock options in Biogen. Catherine Miller is an employee of and holds stock/stock options in Biogen. Andrew Chan has received compensation for activities with Actelion,
Almirall, Bayer HealthCare, Biogen, Celgene, Genzyme, Merck, Novartis, Roche, Sanofi-Aventis, and Teva Neuroscience, all for university research funds; research support from Biogen, Genzyme, and UCB.

Compliance with Ethics Guidelines. The protocol was approved by the relevant Institutional Review Board for each study site. All procedures performed in studies involving human participants were in accordance with the ethical standards of the institutional and/or national research committee and with the 1964 Helsinki declaration and its later amendments or comparable ethical standards. Informed consent was obtained from all individual participants included in the study. The study was conducted in accordance with the Declaration of Helsinki, International Conference on HarmonizationGood Clinical Practice, and all applicable laws and regulations. A waiver of informed consent, based on non-personally identifiable retrospective data collection and minimal risk, was at the discretion of the individual Institutional Review Boards or Ethics Committees for each site. When required, participants provided written informed consent before study enrollment.

Data Availability. The datasets used and/or analyzed during the current study are available from the corresponding author upon reasonable request.

Open Access. This article is distributed under the terms of the Creative Commons Attribution-NonCommercial 4.0 International License (http://creativecommons.org/licenses/ by-nc/4.0/), which permits any noncommercial use, distribution, and reproduction in any medium, provided you give appropriate credit to the original author(s) and the source, provide a link to the Creative Commons license, and indicate if changes were made.

\section{REFERENCES}

1. Giannetti P, Niccolini NR. BG-12 and its potential for the prevention of relapse in multiple sclerosis. Degener Neurol Neuromuscul Dis. 2012;10:119. 
2. Edwards KRPN, Rogge M, Sheikh S, Zhu B. A pharmacokinetic study of delayed-release dimethyl fumarate to evaluate cerebrospinal fluid penetration in patients with secondary progressive multiple sclerosis. Mult Scler. 2016;22[Supp 3]:784-5.

3. Brennan MS, Matos MF, Li B, et al. Dimethyl fumarate and monoethyl fumarate exhibit differential effects on KEAP1, NRF2 activation, and glutathione depletion in vitro. PLoS One. 2015;10(3): e0120254.

4. Fox RJ, Miller DH, Phillips JT, et al. Placebo-controlled phase 3 study of oral BG-12 or glatiramer in multiple sclerosis. N Engl J Med. 2012;367(12): 1087-97.

5. Gold R, Arnold DL, Bar-Or A, et al. Long-term effects of delayed-release dimethyl fumarate in multiple sclerosis: interim analysis of ENDORSE, a randomized extension study. Mult Scler. 2017;23(2):253-65.

6. Gold R, Kappos L, Arnold DL, et al. Placebo-controlled phase 3 study of oral BG-12 for relapsing multiple sclerosis. N Engl J Med. 2012;367(12): 1098-107.

7. Phillips JT, Selmaj K, Gold R, et al. Clinical significance of gastrointestinal and flushing events in patients with multiple sclerosis treated with delayed-release dimethyl fumarate. Int J MS Care. 2015;17(5):236-43.

8. Fox EJ, Vasquez A, Grainger W, et al. Gastrointestinal tolerability of delayed-release dimethyl fumarate in a multicenter, open-label study of patients with relapsing forms of multiple sclerosis (MANAGE). Int J MS Care. 2016;18(1):9-18.

9. Livingston T, Fay M, Iyer R, Wells W, Pill MW. Quantifying differences in health care consumption for the management of multiple sclerosis within privately and publicly insured health care programs. J Manag Care Spec Pharm. 2016;22(12): 1385-91.

10. Phillips JT, Erwin AA, Agrella S, et al. Consensus management of gastrointestinal events associated with delayed-release dimethyl fumarate: a Delphi study. Neurol Ther. 2015;4(2):137-46.

11. Phillips J, Agrella S, Fox RJ. Dimethyl fumarate: a review of efficacy and practical management strategies for common adverse events in patients with multiple sclerosis. Int $\mathrm{J}$ MS Care. 2017;19(2):74-83.

12. Ontaneda D, Vollmer B, Sillau S, et al. Comparative efficacy and discontinuation of fingolimod and dimethyl fumarate in two large academic medical centers. Neurology. 2016;86[Suppl 16]:P3.109.

13. Vu N, Bradshaw M, Moses H, Sriram S, Pawate S. Efficacy and tolerability of fingolimod, dimethyl fumarate, and teriflunomide in patients with multiple sclerosis: real world experience from a single center. Neurology. 2016;86[Suppl 16]:P6168.

14. Biogen. Data on file. Biogen: Cambridge, MA

15. Begus-Nahrmann Y, Niemczyk G, Schmid B, Mäurer $M$. The potential of individualized patient coaching to optimize treatment with delayed-release dimethyl fumarate: a retrospective analysis of patients with multiple sclerosis treated in a real-world setting. Mult Scler. 2016;22[Suppl 3]:636.

16. ClinicalTrials.gov. Observational study to characterize real-world clinical outcomes with relapsingremitting multiple sclerosis (RRMS) (EFFECT) 2016. Updated 23 March 2017. https://clinicaltrials.gov/ ct2/show/NCT02776072.

17. Polman $\mathrm{CH}$, Reingold SC, Banwell B, et al. Diagnostic criteria for multiple sclerosis: 2010 revisions to the McDonald criteria. Ann Neurol. 2011;69(2): 292-302.

18. Gold R, Schlegel E, Elias-Hamp B, et al. Incidence and mitigation of gastrointestinal events in patients with relapsing-remitting Multiple Sclerosis receiving delayed-release dimethyl fumarate-a German phase IV study (TOLERATE). Ther Adv Neurol Disord. 2018;11:1756286418768775.

19. Sammarco C, Laing L, Minetti J, Desanctis C, Herbert J. Strategies to reduce adverse events related to oral dimethyl fumarate. Mult Scler. 2014;20[Suppl 1]:206.

20. Vorobeychik G, Potts J, Smith M, Vlaicu M. A retrospective analysis of discontinuation rates and reasons for discontinuation in multiple sclerosis patients treated with delayed-release dimethyl fumarate (Biogen ONE Support Program). Mult Scler. 2016;22[Suppl 3]:301-2. 